\title{
Geomagnetic Variations in the British Isles
}

\author{
R.N. EDWARDS \& L.K. LAW \\ Department of Geodesy and Geophysics, University of Cambridge
}

(Received January 12, 1970)

\begin{abstract}
The results and the possible interpretation of a survey are described. A comprehensive study of the temporal variations of the three components of the geomagnetic field when measured from place to place has been undertaken using eight magnetometers. A total of forty six temporary magnetic observatories were established covering the majority of Wales, Ireland, Southern Scotland \& England.

The temporal variations of the horizontal components of the geomagnetic field are very similar over the wole area. Variations of periods in the range of four hours to ten minutes were observed.

The temporal variations of the vertical component differ when measured at stations only $40-50 \mathrm{~km}$ apart. On translating the data into the frequency domain, it was observed that variations with a frequency of about 36 cycles per day are most affected.

At nearly all stations, there is a strong correlation between the variations of the vertical field and the variations of some component of the horizontal field. It is possible to interpret this behaviour as indicating the presence of local current concentrations in the vicinity of the British Isles. At first sight, the shallow seas and the Atlantic Ocean appear to be the major conductors. A possible interpretation, consistent with the observations, is that the current concentrations are a direct result of electromagnetic induction in the Atlantic Ocean by the variations of an external magnetic field.

Another current concentration, of similar magnitude to the currents in the seas, is observed to be flowing in a NE-SW direction through Southern Scotland. It is possible that this current is driven by electric potentials set up by the current concentrations in the Irish and North Seas. It is suggested that it may not be necessary to postulate the existence of an upper mantle or crustal conductivity anomaly in this region.
\end{abstract}

